\title{
A Robust Planning System for Agricultural Management: Modelling and Algorithm
}

\author{
Bahram Alidaee ${ }^{1}$, Haibo Wang ${ }^{2}$ \\ ${ }^{1}$ University of Mississippi, School of Business \\ University, MS 38655, USA \\ Balidaee@bus.olemiss.edu \\ ${ }^{2}$ The Sanchez School of Business, Texas A\&M International University \\ Laredo, TX 78041 \\ Hwang@tamiu.edu
}

\section{Extended Abstract}

In the harvest season, farms will hire many of temporary (seasonal) workers to harvest the crops with a time window due to weather condition and market price. During the harvest season, there are multiple machines working on the same field and these machines can be controlled by one worker or multiple workers depending on the system in the machines. If they are driverless vehicles, one worker can manage several machines in the same time, [1]. We propose an integer programming model based Capacitated Facility Location Problem (CFLP) for handling seasonal workers and robotic systems and solve the problem with an effective heuristic. The heuristic is a hybrid of Genetic Algorithm (GA) and Tabu Search (TS). The TS is based on Critical Even Memory Tabu Search and the GA works as an effective diversification strategy within the TS. Our model can be effective in many combinatorial optimization problems, including quadratic assignment problem, uncapacitated facility location, uncapacitated p-median problems, multi-machine scheduling problems, [2], [3], [4], and [5]. Comprehensive computational experiment will be provide to show effectiveness of the hybrid algorithm.

\section{References}

[1] Agricultural Robotics \& Automation. [Online]. Available: http://www.ieee-ras.org/agricultural-robotics-automation

[2] B. Alidaee, and H. Wang. "A note on heuristic approach based on UBQP formulation of the maximum diversity problem," JORS, vol. 68, pp. 102-110, 2017.

[3] B. Alidaee, H. Sloan, and H. Wang. "Simple and fast novel diversification approach for the UBQP based on sequential improvement local search,” C\&IE, vol. 111, pp. 164-175, 2017.

[4] B. Alidaee, and H. Wang, "A hybrid genetic-tabu search for an extension of uncapacitated facility location problem," submitted.

[5] B. Alidaee, and H. Wang, "Unrelated parallel machine selection and job scheduling with the objective of minimizing total workload and machine fixed costs," IEEE Transactions on Automation Science and Engineering, accepted, 2017. 